\title{
Laparoscopic Diagnosis and Excision of a Giant Sigmoid Cystic Duplication
}

\author{
Mirko Bertozzi, MD, and Antonino Appignani, MD, PhD \\ S.C. di Clinica Chirurgica Pediatrica, Università degli Studi di Perugia, Ospedale S. Maria della Misericordia, \\ Perugia, Italy (all authors).
}

\begin{abstract}
The authors report a very rare case of giant duplication of sigmoid colon in a 4-year-old girl successfully diagnosed and treated with a laparoscopy-assisted technique. This case shows that laparoscopy is useful in definitive diagnosis of giant cystic masses with a preoperative undiagnosed origin. Laparoscopy-assisted treatment of these benign masses may be realized, even in difficult cases such as the one described.
\end{abstract}

Key Words: Enteric duplications, Abdominal cyst, Sigmoid colon, Pediatric age.

Citation Bertozzi M, Appignani A. Laparoscopic diagnosis and excision of a giant sigmoid cystic duplication. CRSLS e2014.00238. DOI: 10.4293/CRSLS.2014.00238.

Copyright (C) 2014 SLS This is an open-access article distributed under the terms of the Creative Commons Attribution-Noncommercial-ShareAlike 3.0 Unported license, which permits unrestricted noncommercial use, distribution, and reproduction in any medium, provided the original author and source are credited.

Address correspondence to: Mirko Bertozzi, M.D. S.C. di Clinica Chirurgica Pediatrica, Università degli Studi di Perugia, Ospedale S. Maria della Misericordia Loc. S. Andrea delle Fratte, 06100 Perugia, Italy. Telephone: (+39) 075-578-3376, Fax: (+39) 075-578-3376; E-mail: mirkobertozzi@hotmail.com

\section{INTRODUCTION}

Duplications of the alimentary tract include a wide variety of mass lesions throughout the course of the gastrointestinal tract that are either tubular or cystic. Duplications of the colon and rectum constitute about $17 \%$ of all enteric duplications and may be distinguished in 3 general classes: cystic or short tubular duplications that reside in the mesentery of the colon, duplications that reside in the midline, and side-to-side duplications that can vary in length and location. ${ }^{1}$ Complete resection of a colonic duplication is necessary because of potential malignant transformation.

\section{CASE DESCRIPTION}

A 4-year-old girl was admitted to our hospital with abdominal pain of 3 days' duration. On admission, she admitted that symptoms had increased in intensity over the last day.

The physical examination was unremarkable except for the abdominal examination. The abdomen was slightly protuberant and there was marked tenderness localized in the mid and lower abdominal quadrants. All laboratory findings were normal.

An ultrasonographic scan showed a large cystic mass occupying the mid and lower quadrant of the abdomen. A computed tomographic scan of the abdomen showed a $12 \times 16-\mathrm{cm}$ cystic mass of uncertain origin (Figure 1). Tumor markers were normal.

Laparoscopy was performed with the patient in the supine position (Figure 2). An open method was used to introduce the first 5-mm port in the right hypochondrium because of the big dimension of the mass. The abdomen was insufflated with carbon dioxide with a pressure of $10 \mathrm{~mm} \mathrm{Hg}$ and flow of $1 \mathrm{~L} / \mathrm{min}$. Laparoscopic exploration of the abdominal cavity immediately revealed the cyst. A second operative 5-mm port was inserted into the right flank. The cyst was then mobilized with an atraumatic grasper and its relationship with the sigmoid colon was individuated (Figure 3). An intravenous 16-gauge catheter was inserted through the abdominal wall at the umbilical level and under direct laparoscopic vision through the wall of the cyst. Aspiration of cyst content was performed, avoiding spillage by suction of $>500 \mathrm{~mL}$ of mucoid fluid until the cyst was completely drained. The needle was withdrawn and the specimen was delivered under direct laparoscopic vision through a $10-\mathrm{mm}$ additional umbilical incision to the abdominal surface for extracorporeal resection (Figure 4). A $4-\mathrm{cm}$ sigmoid colon resection and an end-to-end anastomosis were performed (Figure 5). Once the bowel was reintroduced into the abdominal cavity, laparoscopic check of the anas- 


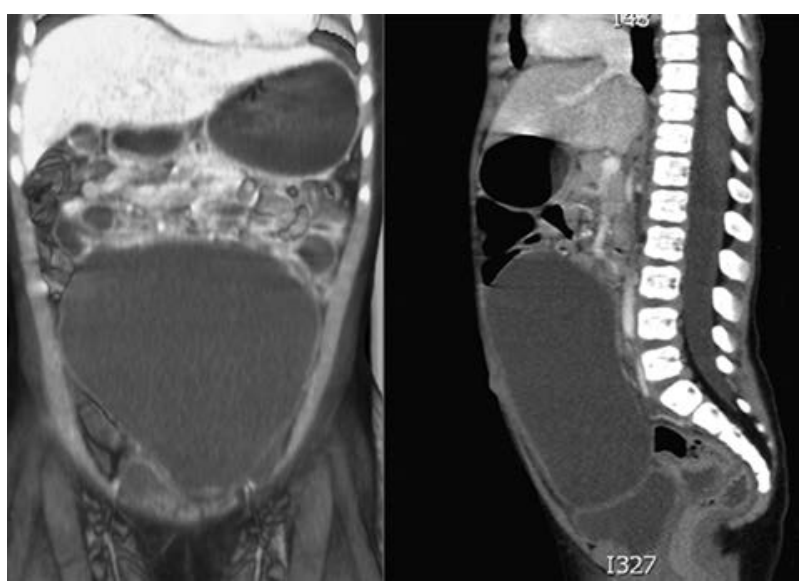

Figure 1. Computed tomographic scan images demonstrating the giant abdominal cystic mass of undiagnosed origin.

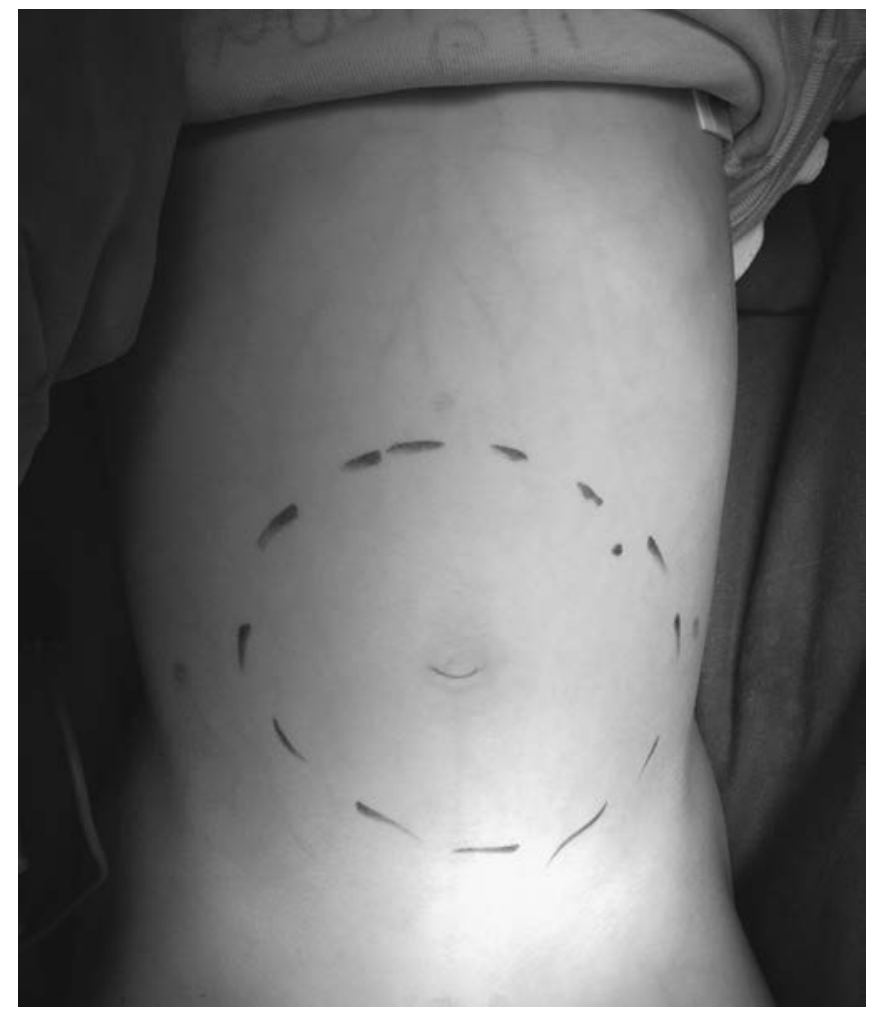

Figure 2. Limits of the mass in a preoperative image.

tomosis, hemostasis, and interval appendectomy were accomplished. The postoperative course was uneventful.

Histology results confirmed the diagnosis of side-to-side sigmoid duplication (Figure 6).

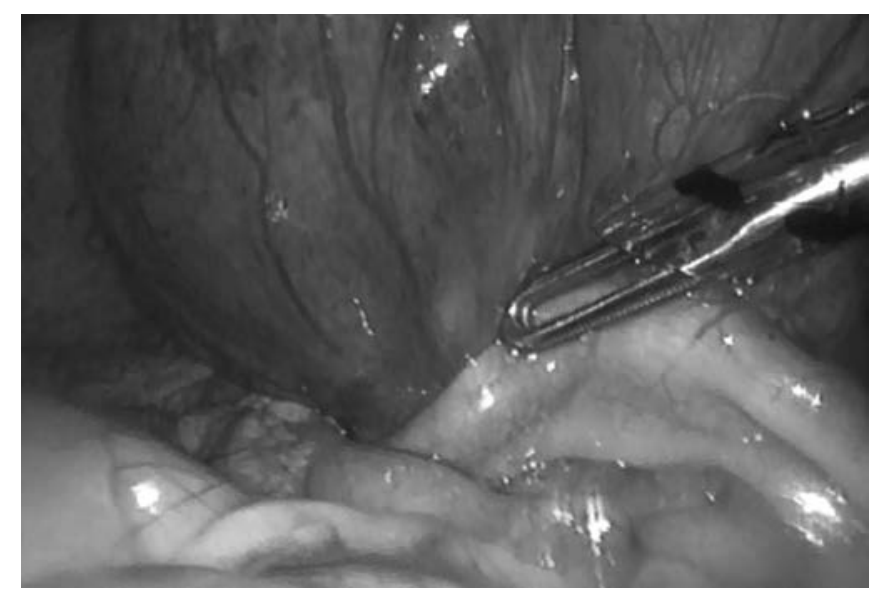

Figure 3. Laparoscopic operative image: the cyst is mobilized with an atraumatic grasper and its sigmoid relationship is clearly individuated.

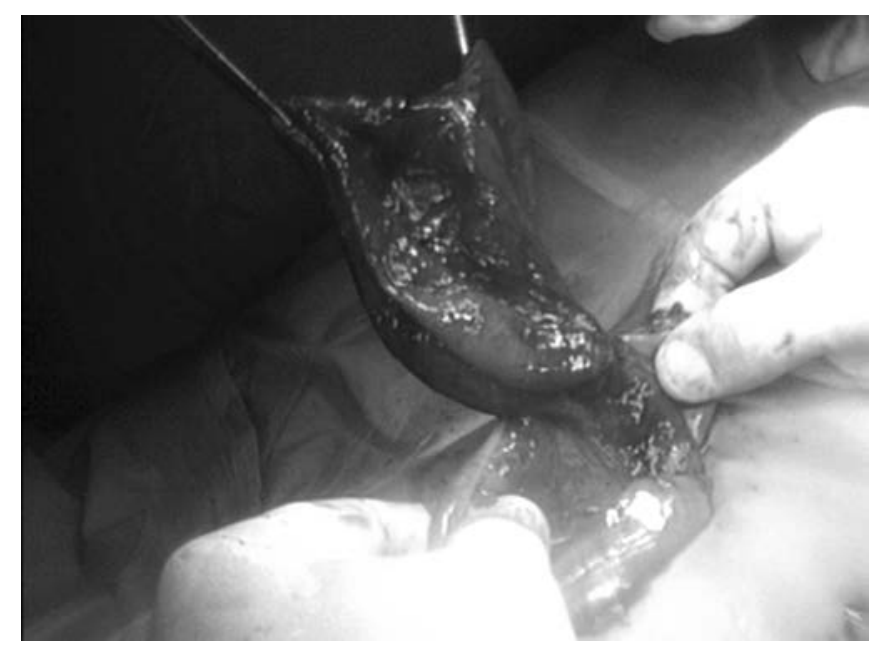

Figure 4. The collapsed sigmoid duplication is delivered through a $10-\mathrm{mm}$ additional umbilical incision to the abdominal surface for extracorporeal resection.

\section{DISCUSSION}

Intestinal duplications are rare congenital abnormalities. The clinical presentation of these malformation ranges from infancy to adulthood. ${ }^{2-5}$ Most cases (from 70\%-80\%) present within the first year of life, ${ }^{2-5}$ even if intestinal duplications are becoming more frequently identified in utero with the use of prenatal ultrasonography The signs and symptoms depend on the type and location of the duplication. Infants and neonates usually present with vomiting, abdominal distention, pain, or an abdominal mass. Less frequently, vomiting, diarrhea, and rectorrhagia are present.2,5 Rarely, complications such as volvulus, intussusception perforation, and bleeding from ulceration may occur.,2,3 


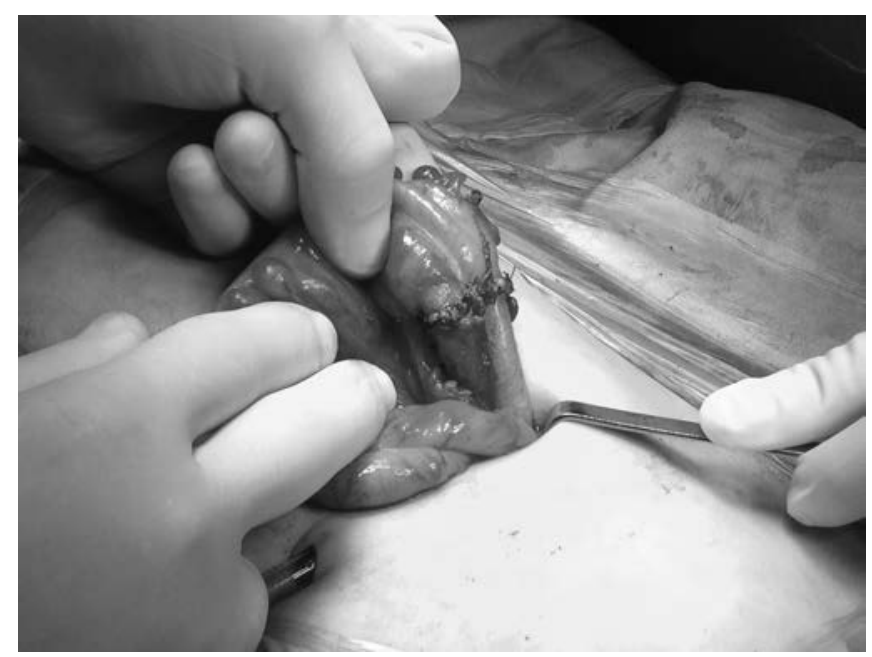

Figure 5. After a 4-cm sigmoid colon extracorporeal resection, including the duplication, an end-to-end anastomosis is performed.

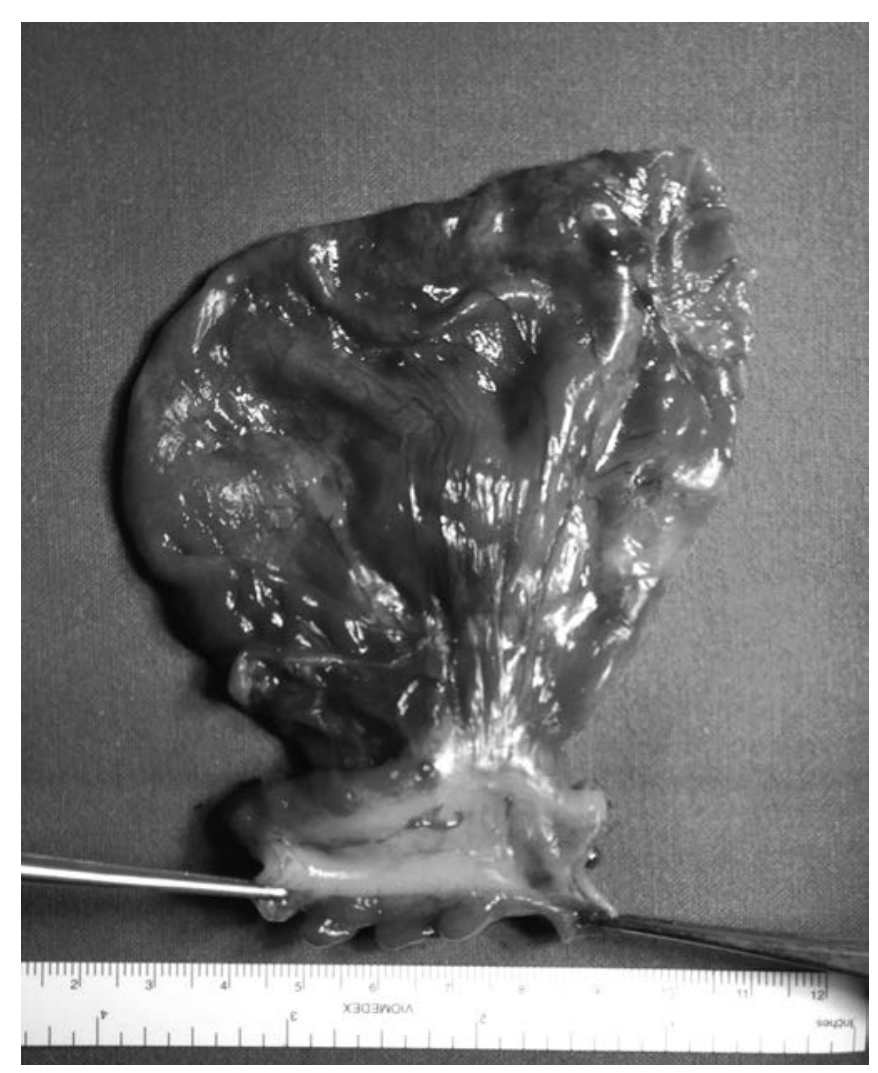

Figure 6. The excised sigmoid colon including the side-to-side cystic duplication.

Duplications of the colon are rare, with fewer than 100 cases of colonic duplication reported in the literature. ${ }^{6}$ In a recent series, these abnormalities represent only $6.8 \%$ of all gastrointestinal duplications. ${ }^{3}$
Duplications of the colon and rectum may be clinically silent, and most colonic duplication cysts remain asymptomatic and may remain undiagnosed until adulthood.

When symptomatic, abdominal pain is the most common presentation. ${ }^{7}$ Other symptoms of this kind of duplication are constipation, obstruction, or abdominal distention. ${ }^{2,5}$ Malignant degeneration is a rare but well-known occurrence. ${ }^{8-10}$

The treatment of symptomatic colonic duplications is en bloc resection of the cyst and adjacent viscera. Sometimes small cystic duplications can be excised without colonic resection if blood flow to the adjacent intestinal segment is not compromised.

There are few reports of laparoscopic or laparoscopy-assisted treatment of the alimentary tract duplication in the pediatric English literature ${ }^{11-14}$; among these, only two report colorectal involvement. ${ }^{15,16}$ These 2 cases describe the excision of tubular duplications. The case described by Chang et $\mathrm{al}^{15}$ is a T-shaped duplication of descending colon in a 7-year-old girl. After the entire duplication was mobilized using a 3-port transperitoneal laparoscopic technique, the authors delivered the specimen through an additional left upper quadrant incision to the abdominal surface for extracorporeal resection. Park et $\mathrm{al}^{16}$ performed a laparoscopic resection of a tubular duplication of the sigmoid colon under the guidance of an intraoperative colonoscopy in an 8-yearold girl. In this case, the resection of the duplication was achieved entirely by the means of laparoscopy with a 12-mm endoscopic GIA stapler.

In the case of giant cystic masses in females with apparent pelvic origin, the differential diagnosis includes enteric duplications, ovarian cysts, mesenteric cysts, and lymphangiomas.

If conventional radiologic examinations including ultrasonography and computed tomography scan fail to assess the exact origin of a cystic mass because of its unusually large size, the final diagnosis may be confirmed only during intervention. In these cases, laparoscopy may be useful.

In the case of giant cysts crossing the transverse umbilical line, as in the case under discussion, it is possible to move into the upper quadrants with the usual placement of the first umbilical trocar gaining working space. The first 5-mm trocar for the telescope was placed in the right hypochondrium, with an additional operative 5-mm trocar placed in the right flank to mobilize and assess the cyst's origin. In this manner, even though the cyst was very big and filled the abdominal cavity, it was very easy to assess its sigmoid origin and, under direct laparoscopic vision, to 
perform transabdominal aspiration, avoiding spillage until the cyst was completely drained.

Extracorporeal 4-cm sigmoid resection including the cyst and an end-to-end anastomosis was performed exteriorizing the empty cyst through an additional $1-\mathrm{cm}$ incision at the umbilical scar level, giving excellent cosmetic results.

To our knowledge, this is the first case reported of giant side-to-side cystic duplication of sigmoid colon in a child successfully treated by laparoscopy-assisted resection.

\section{References:}

1. Coran AG, Adzick NS, Krummel TM, Laberge JM, Shamberger RC, Caldamone AA. Pediatric Surgery, vol. 2. 7th ed. Philadelphia: Elsevier-Saunders; 2012.

2. Ashcraft KW, Holcomb III GW, Murphy JP. Pediatric Surgery. 4th ed. Philadelphia: Elsevier-Saunders; 2005.

3. Puligandla PS, Nguyen LT, St-Vil D, et al. Gastrointestinal duplications. J Pediatr Surg. 2003;38:740-744.

4. Stern LE, Warner BW Gastrointestinal duplications. Semin Pediatr Surg. 2000;9:135-140.

5. Iyer CP, Mhour GH. Duplications of the alimentary tract in infants and children. J Pediatr Surg. 1995;30:1267-1270.

6. Fotiadis C, Genetzakis M, Papandreou I, Misiakos EP, Agapitos E, Zografos GC. Colonic duplication in adults: report of two cases presenting with rectal bleeding. World J Gastroenterol. 2005;11:5072-5074.

7. Choong CK, Frizelle FA. Giant colonic diverticulum: report of four cases and review of the literature. Dis Colon Rectum. 1998;41:1178-1185.
8. Horie H, Iwasaki I, Takahashi H. Carcinoid in a gastrointestinal duplication. J Pediatr Surg. 1986;21:902-904.

9. Inoue $\mathrm{Y}$, Nakamura $\mathrm{H}$. Adenocarcinoma arising in colonic duplication cysts with calcification: CT findings of two cases. Abdom Imaging. 1998;23:135-137.

10. Hickey WF, Corson JM. Squamous cell carcinoma arising in a duplication of the colon: case report and literature review of squamous cell carcinoma of the colon and of malignancy complicating colonic duplication. Cancer. 1981;47:602-609.

11. Schalamon J, Schleef J, Höllwarth ME. Experience with gastro-intestinal duplications in childhood. Langenbecks Arch Surg. 2000;385:402-405.

12. Kohno M, Ikawa H, Konuma K, et al. Laparoscopic enucleation of a gastroenteric duplication cyst arising in a pancreatic tail that did not communicate with the pancreatic duct: report of a case. Surg Today. 2010;40:281-284.

13. Pal K, El Shafei H, Al Buainain H, Mitra DK. Successful laparoscopic treatment of hemorrhage from ileal duplication cyst in a 10-year-old Saudi boy. J Laparoendosc Adv Surg Tech A. 2010;20:99-101.

14. Kim YJ, Kim YK, Jeong YJ, Moon WS, Gwak HJ. Ileal duplication cyst: Y-configuration on in vivo sonography. $J$ Pediatr Surg. 2009; 44:1462-1464.

15. Chang YT, Lee JY, Liao YM, Chiou SS. Laparoscopic resection of a giant retroperitoneal T-shaped duplication of descending colon. J Pediatr Surg. 2008;43:401-404.

16. Park YA, Jung EJ, Han SJ. Laparoscopic resection of duplicated sigmoid colon under the guidance of intraoperative colonoscopy. Surg Laparosc Endosc Percutan Tech. 2005;15: 299-301. 